Article

\title{
Counterion Condensation and Effective Charge of PAMAM Dendrimers
}

\section{Ute Böhme, Anja Klenge, Brigitte Hänel and Ulrich Scheler *}

Leibniz-Institut für Polymerforschung Dresden e.V., Hohe Str. 6, 01069 Dresden, Germany; E-Mails: boehme-ute@ipfdd.de (U.B.); anja.klenge@web.de (A.K.); haenel@ipfdd.de (B.H.)

* Author to whom correspondence should be addressed; E-Mail: scheler@ipfdd.de; Tel.: +49-351-4658-275; Fax: +49-351-4658-231.

Received: 24 March 2011 / Accepted: 14 April 2011 / Published: 27 April 2011

\begin{abstract}
PAMAM dendrimers are used as a model system to investigate the effects of counterion condensation and the effective charge for spherical polyelectrolytes. Because of their amino groups, PAMAM dendrimers are weak polyelectrolytes. Lowering the $\mathrm{pH}$ results in an increasing protonation of the amino groups which is monitored via the proton chemical shifts of the adjacent $\mathrm{CH}_{2}$ groups. The effective charge is determined from a combination of diffusion and electrophoresis NMR. The fraction of the charges, which are effective for the interaction with an external electric field or other charges, decreases with increasing generation (size) of the dendrimers.
\end{abstract}

Keywords: effective charge; dendrimers; spherical polyelectrolytes; electrophoresis NMR

\section{Introduction}

The electrostatic interaction between charged molecules is an important interaction in materials and in biological systems. Counterions condense on highly charged macromolecules, because the electric field generated by the highly charged macromolecules is too strong, so that a counterion could escape it based on its thermal energy [1]. Condensed counterions lower the charge density and thus reduce the electric field, so that the remaining counterions can escape the electric field generated from the macromolecule including the condensed counterions. Counterion condensation has initially been described for linear polyelectrolytes. 
Counterion condensation has been observed directly using electrophoresis NMR [2]. Electrophoresis NMR [3] in conjunction with diffusion NMR [4] provides the opportunity to determine the effective charge of molecules without any model [5]. On the timescale of the NMR experiment charged molecules have a constant velocity resulting from the force balance between the driving force in the electric field and the hydrodynamic friction. From that force balance the effective charge is calculated according to Equation 1

$$
z=\frac{\mu \cdot k_{B} \cdot T}{e \cdot D}
$$

where $\mu$ is the electrophoretic mobility, $\mathrm{D}$ the diffusion coefficient, $\mathrm{T}$ the temperature, $\mathrm{k}_{\mathrm{B}}$ the Boltzmann constant and e the elementary charge.

With the knowledge of the nominal charge, the degree of counterion condensation is inferred. This approach has been applied extensively to linear polyelectrolytes, mostly poly(styrene sulfonate), a discussion of the influences from the solvent properties like ionic strength or dielectric constant is found in [6] and in references therein. Variation of the kind of counterion shows, that there are ion-specific effects as well [7].

Because counterion condensation has initially been derived for linear polyelectrolytes, there is a fundamental interest in the generalization of that effect to globular molecules. First examples have been demonstrated on spherical polyelectrolyte brushes [8,9] and proteins [10]. Dendrimers have been chosen as a model, because they are well characterized in size and structure and they contain only two kinds of ionizable groups.

A number of simulations exists [11-15], studying the variation of $\mathrm{pH}$ or electrostatic interactions in particular on structure and dynamics of dendrimers. The influence of counterion condensation for generic dendrimer with a three functional core (as opposed to PAMAM dendrimers, which have a tetrafunctional core) and terminal charged cationic dendrimers has been studied in $[13,14]$. Both observed, that increasing strength of electrostatic interaction provokes counterion condensation, but no effective number of charges has been derived for the typically conditions of dendrimers in aqueous solutions. Simulations of counterion condensation and subsequent calculations of $\zeta$ potential for PAMAM dendrimers of generation 3 to 7 have been conducted by [15]. They found an only slowly increasing $\zeta$ potential (a measure of the electrophoretic mobility in an electric field) with growing generation and saturation at high generation. This is contrary to the exponential behavior of the nominal surface charge, which has been derived from the number of protonated terminal groups and the simulated counterion distribution.

\section{Results and Discussion}

In Figure 1, a series of ${ }^{1} \mathrm{H}$ NMR spectra of a PAMAM dendrimer of generation 2 as a function of the $\mathrm{pH}$ is shown. The shifts of the proton signals from $\mathrm{CH}_{2}$ groups adjacent to the primary and tertiary amino groups are indicated by pink and blue color, respectively. Lowering the $\mathrm{pH}$ in an initial step the primary amino groups are protonated. The primary amino groups are fully protonated at $\mathrm{pH}=7.5$. Subsequently there is a monotonic shift of the proton signal of $\mathrm{CH}_{2}$ groups next to the tertiary amino groups. The monotonic shift shows, that the equilibrium between protonated and unprotonated tertiary amino groups shifts towards the protonated amino groups with fast exchange of the protons. For each 
generation and each $\mathrm{pH}$ the equilibrium between unprotonated and protonated states and thus the nominal charge maybe determined from these spectra.

Figure 1. (a) Branch of a PAMAM dendrimer of generation 2. The methylene groups next to the terminal primary amino groups are labeled in pink and next to the interior tertiary amino groups are labeled with blue color. (b) ${ }^{1} \mathrm{H}$ NMR spectra of PAMAM G2 at different $\mathrm{pH}$ values showing the chemical shift of the selected methylene groups as function of $\mathrm{pH}$ with a label of the same color as in (a).

(a)
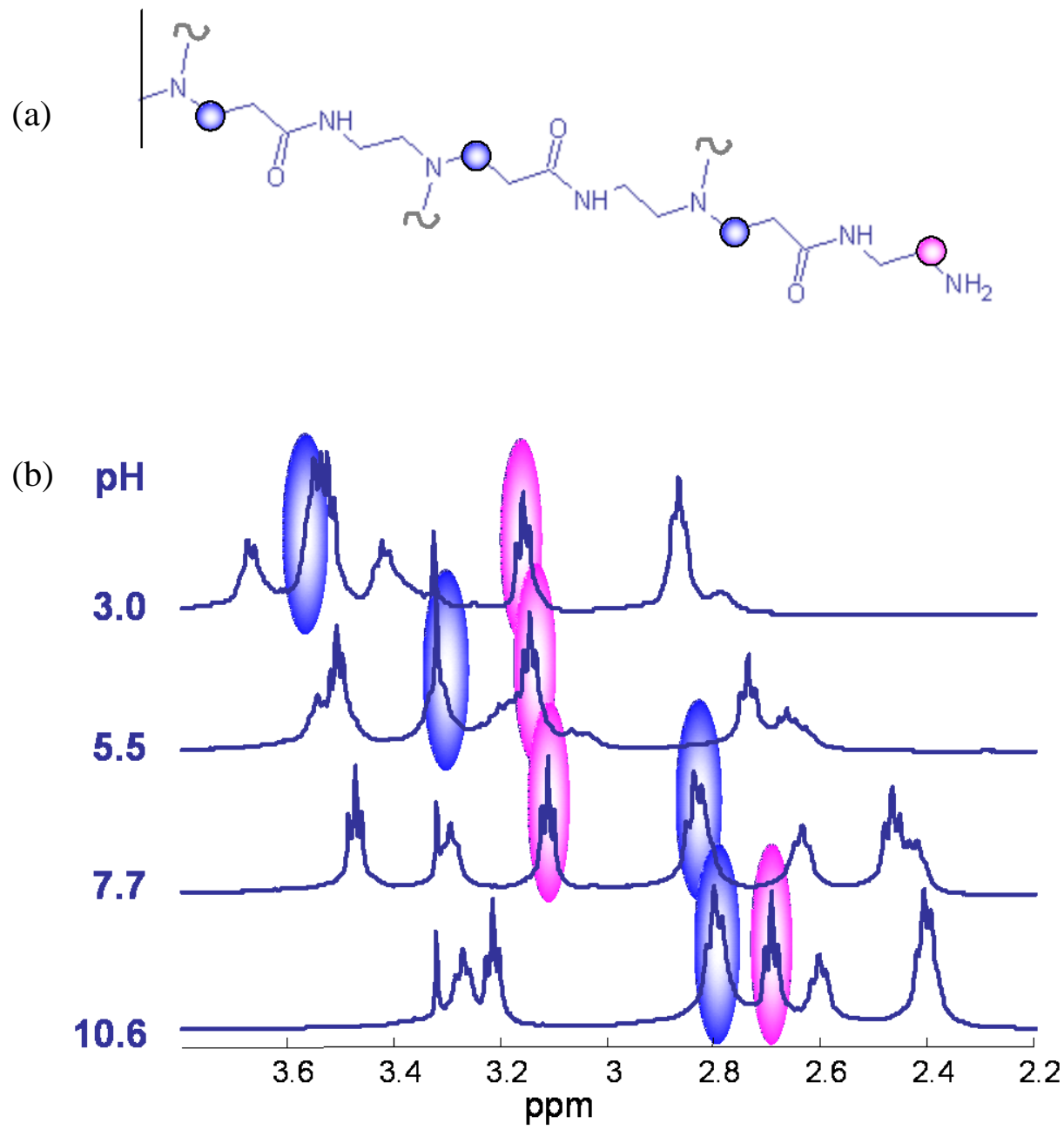

For example in generation 2 all amino groups are unprotonated at $\mathrm{pH}=10.5$. At $\mathrm{pH}=7.7$, all primary amino groups are protonated while the tertiary amino groups are nonprotonated resulting in a nominal charge of 16 . At $\mathrm{pH}=5.5,55 \%$ of the tertiary amino groups are protonated resulting in a total nominal charge of 24 and at $\mathrm{pH}=3$ all amino groups are protonated resulting in the maximum nominal charge of 32 .

Comparable results have been reported from titration experiments [15]. They found, that the protonation of the primary amino groups is completed at $\mathrm{pH} \sim 7$ and the protonation of the tertiary groups except one group of the central ethylenediamine at $\mathrm{pH} \sim 3$.

In Figure 2, an example of signal decay from a diffusion experiment in a Stejskal-Tanner plot [4] is shown. Because the dendrimers are monodisperse, the decay in the Stejskal-Tanner plot is linear. From the slope, the diffusion coefficient is derived according Equation 2. 


$$
\frac{S}{S_{0}}=\exp \left[-D \gamma^{2} \delta^{2} g^{2}\left(\Delta-\frac{\delta}{3}\right)\right]
$$

with $\mathrm{D}$ the diffusion coefficient, $\Delta$ the observation time, $\gamma$ the magnetogyric ratio, $\mathrm{g}$ and $\delta$ the gradient strength and duration respectively.

Figure 2. Stejskal-Tanner plot [4] of signal decay for PAMAM G2 at pH 5.

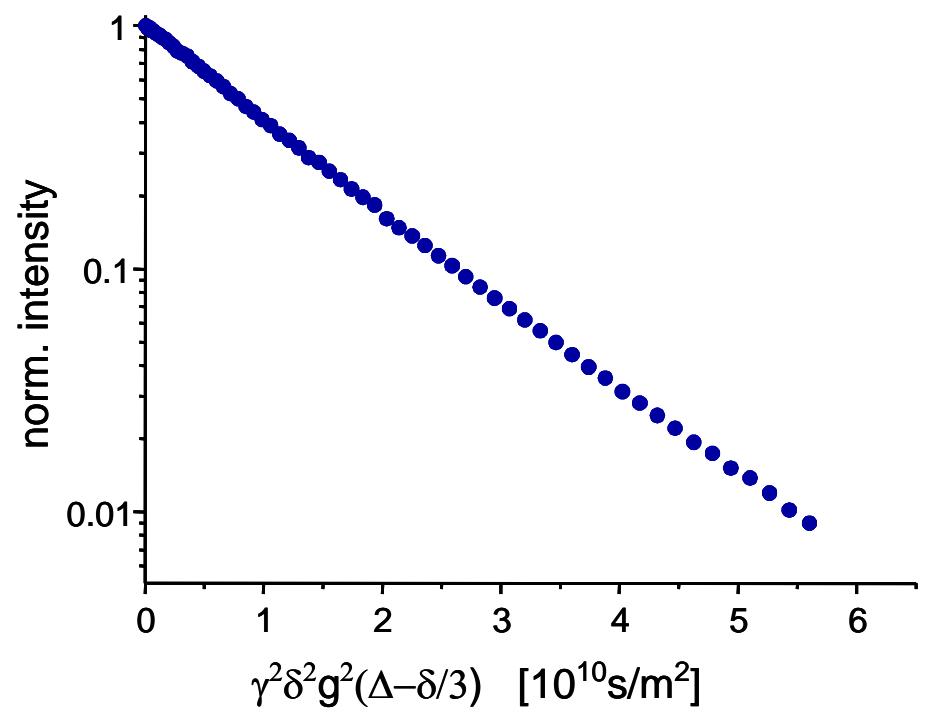

Figure 3 shows a two-dimensional electrophoresis NMR spectrum, which correlates ${ }^{1} \mathrm{H}$ chemical shift with the electrophoretic mobility. To exclude the effect of the superimposed diffusion, in the electrophoresis NMR experiment only the strength of the applied electric field has been incremented in the indirect dimension. To observe the sign of the charge positive and negative electric fields are applied. Data processing based on a two-dimensional Fourier transform yields a pure two-dimensional absorption spectrum correlating chemical shift and electrophoretic mobility [5].

Figure 3. Two-dimensional electrophoresis NMR spectrum of PAMAM G2 at pH 3.4.

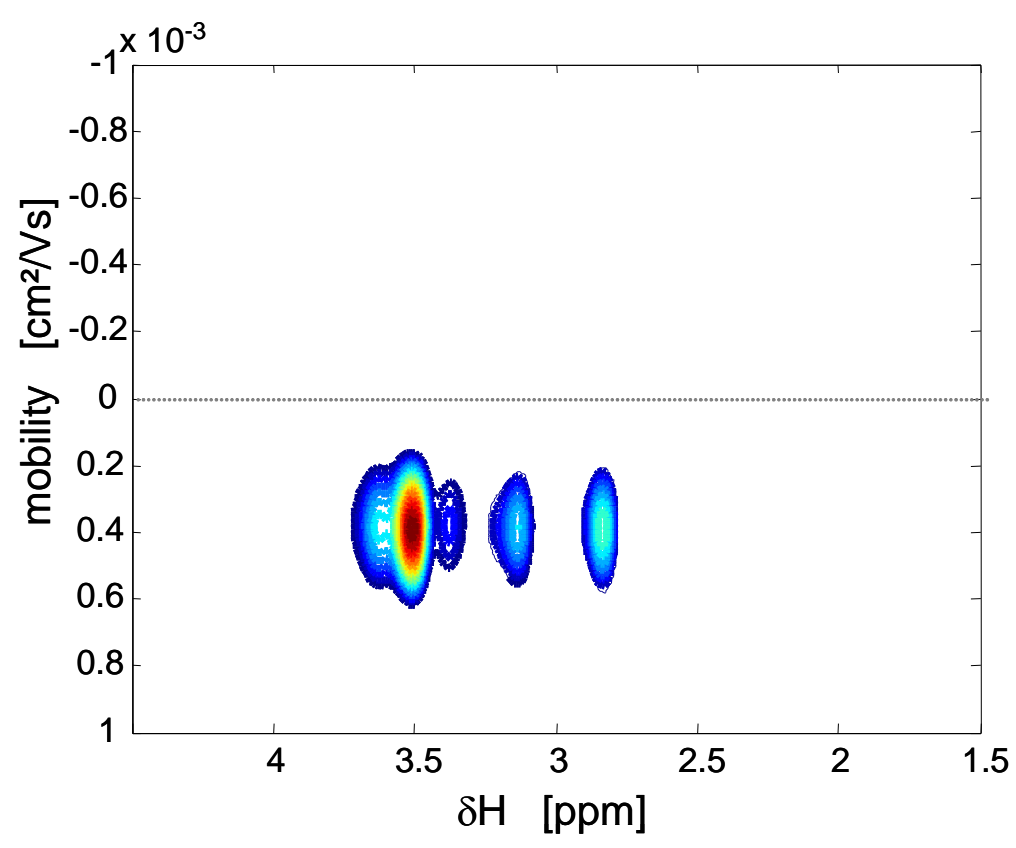


The effective charge together with the nominal charge of the PAMAM dendrimers as a function of their generation are depicted in Figure 4 for two $\mathrm{pH}$ values. On the left at $\mathrm{pH}=7.7$ the primary amino groups are protonated while the tertiary amino groups are non-protonated. On the right at $\mathrm{pH}=3.0$ both the primary and the tertiary amino groups are protonated. This results in a much higher nominal charge for generations 1 and above. However, the effective charge does not increase significantly for any generation, when the $\mathrm{pH}$ is lowered from 7.7 to 3 . This implies, that the maximal effective charge for generation 3 is even lower than the number of primary amino groups.

Figure 4. Comparison of nominal charge (blue) and effective charge (pink) PAMAM dendrimers for generations 0 to 3 . On the left $\mathrm{pH}=7.7$ the primary amino groups are protonated while the tertiary amino groups are unprotonated. On the right $\mathrm{pH}=3$ all amino groups are protonated, the nominal charge is the sum of the primary and tertiary amino groups.
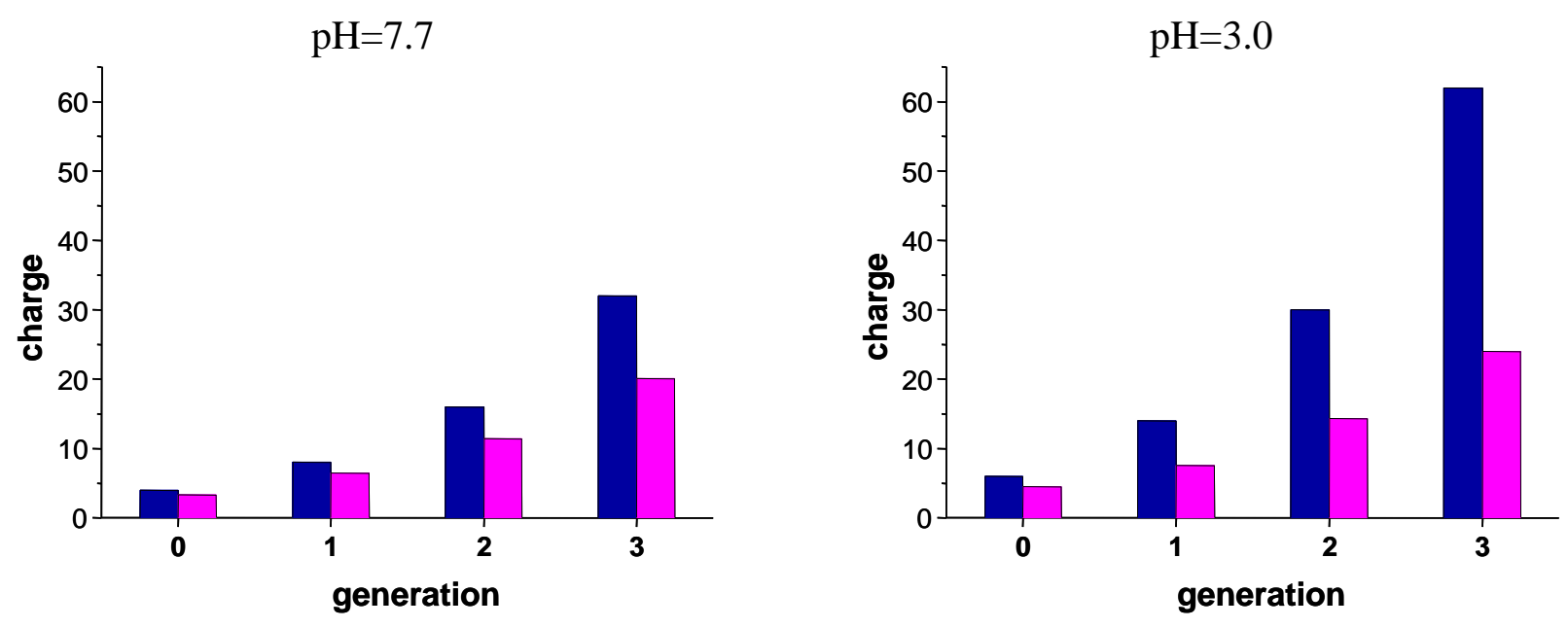

With increasing size (generation) of the dendrimer, there is an increasing deviation of the effective charge from the nominal charge. To illustrate that further, in Figure 5 the ratio between the effective and the nominal charge is shown. This ratio decreases from close to 1 for generation 0 down to 0.4 at higher generations. Counter ion condensation increases with the generation and thus with increasing nominal charge. Chen et al. [16] found in their investigations of PAMAM dendrimers depending on $\mathrm{pH}$ for generation 3 to 6 with SANS the similar trend. They found with increasing addition of DCl upon an amount equal to the full protonation of all primary amino groups of the dendrimer, a rising charge that hardly increased by further protonation. In the full protonated state of the dendrimers the counterion condensation increases with increasing generation, the fraction of the effective charge drops. Their result for generation G3 is in excellent agreement with our investigations. We observe a trend similar to the simulations in [15] for generation 1 to 3 . 
Figure 5. Fraction of effective charge of fully protonated PAMAM dendrimers as a function of their generation.

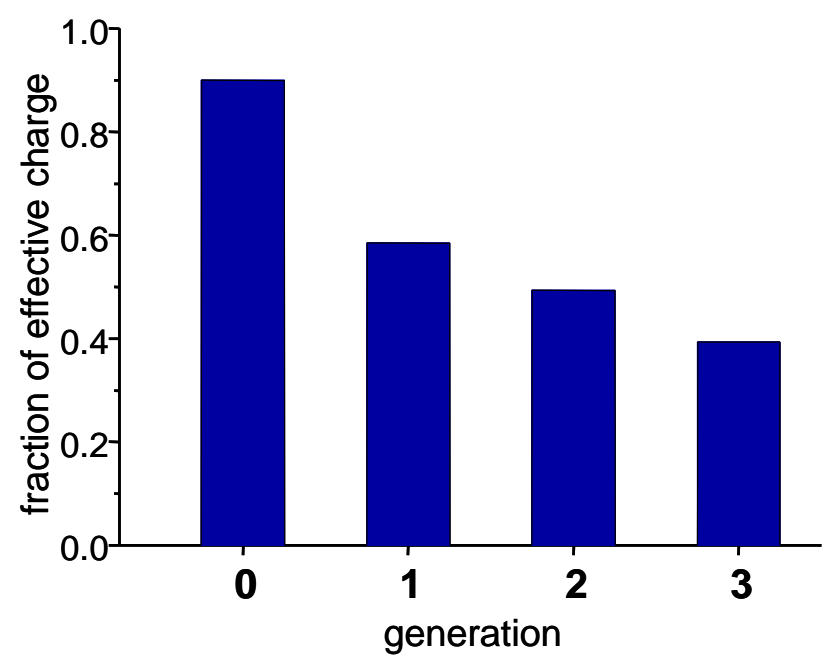

\section{Experimental Section}

The ${ }^{1} \mathrm{H}$ NMR spectra and the diffusion data of PAMAM have been acquired on a Bruker AV 500 NMR spectrometer operating at a Larmor frequency of $500 \mathrm{MHz}$ for ${ }^{1} \mathrm{H}$ using a Diff 30 diffusion NMR probe. The probe generates pulsed magnetic field gradients up to $12 \mathrm{~T} / \mathrm{m}$. For diffusion experiments a stimulated echo sequence with 64 increments in the gradient strength from 0.3 to $9 \mathrm{~T} / \mathrm{m}$ and a diffusion time $\Delta=10 \mathrm{~ms}$ were used.

The electrophoresis NMR experiments have been performed on a Bruker AV 300 NMR spectrometer operating at a Larmor frequency of $300 \mathrm{MHz}$ for protons. The spectrometer is equipped with a Micro 2.5 gradient system generating magnetic field gradients up to $1 \mathrm{~T} / \mathrm{m}$. An in-house-built electrophoresis NMR probe has been used, in which two platinum electrodes are immersed in the sample solution which are connected to a DC amplifier for up to 1,000 V which is connected to one of the gradient channels of the NMR spectrometer. For the measurement of electrophoretic mobility the pulsed field gradient stimulated echo NMR experiment with an applied electric field at duration $\Delta$ has been used. The gradient strength was kept constant at $40 \%$ of the maximum gradient strength and the electric field was incremented in 16 steps from positive max to negative max field strength. The duration $\Delta$ and the maximum field strength have been optimized for each experiment depending on the diffusion behavior of the sample and the conductivity due to added acid.

PAMAM dendrimers with ethylenediamine core and primary amino surface groups of generation 0 to 3, dissolved in methanol, have been purchased from Aldrich. The samples have been concentrated in a rotary evaporator and evacuated afterwards to remove methanol. For all NMR diffusion and electrophoresis experiments a concentration of $1 \mathrm{~g} / \mathrm{L}$ PAMAM in deuterated water $\left(\mathrm{D}_{2} \mathrm{O}, 99.9 \%\right.$, Deutero $\mathrm{GmbH}$ ) has been used. For the investigation of the chemical shift as a function of the $\mathrm{pH}$ the concentration was $20 \mathrm{~g} / \mathrm{L}$ in $\mathrm{D}_{2} \mathrm{O}$. The peak of the residual methyl groups of methanol has been used as internal standard and calibrated to $3.31 \mathrm{ppm}$. The assignment of the proton signals to the PAMAM methylene groups has been derived from dedicated 2D-Spectra (COSY, ${ }^{1} \mathrm{H}-{ }^{13} \mathrm{C}$ HMBC) and from the literature [17]. The $\mathrm{pH}$ was adjusted with deuterium chloride ( $\mathrm{DCl}, 7.6 \mathrm{~N}$ in $\mathrm{D}_{2} \mathrm{O}, 99 \% \mathrm{D}$, Euriso-top). 
The $\mathrm{pH}$ values were measured with a conventional Sartorius PP-20/PY-P11 pH meter. The differences between $\mathrm{pH}$ and $\mathrm{pD}$ were not considered. All experiments were conducted at $20^{\circ} \mathrm{C}$.

\section{Conclusions}

It has been shown, that the effective charge of spherical polyelectrolytes is much lower than the nominal charge, PAMAM dendrimers have been used as an example. The amino groups present in the core and as terminal groups render the dendrimers a weak polyelectrolyte. The degree of protonation, and thus the nominal charge, is determined by ${ }^{1} \mathrm{H}$ NMR spectroscopy as a function of the $\mathrm{pH}$. The effective charge has been determined by a combination of diffusion and electrophoresis NMR.

While the PAMAM dendrimers have negligible charge at their natural $\mathrm{pH}$, the protonation, and thus the nominal charge, increases, lowering the $\mathrm{pH}$ down to 3 , where the primary amino groups are protonated first and subsequently the tertiary amino groups. In any case the effective charge does not increase below $\mathrm{pH}$ 6. For the fully protonated dendrimers the fraction of charges, which are compensated by condensed counterions, increases with the generation of the dendrimers up to $60 \%$ for generation 3 .

\section{Acknowledgements}

The authors would like to thank Hartmut Komber for assistance in the assignment of the ${ }^{1} \mathrm{H}$ NMR spectra of the PAMAM dendrimers.

\section{References}

1 Manning, G.S. Limiting laws for equilibrium and transport properties of polyelectrolyte solutions. In Polyelectrolytes; Sélégny, E., Mandel, M., Strauss, U.P., Eds.; D. Reidel Publishing Company: Dordrecht, The Netherlands, 1974; pp. 9-34.

2 Böhme, U.; Scheler, U. Counterion mobility and effective charge of polyelectrolytes in solution. Macromol. Symp. 2004, 211, 87-92.

3 Holz, M. Electrophoretic NMR. Chem. Soc. Rev. 1994, 23, 165-174.

4 Stejskal, E.O.; Tanner, E.J. Spin diffussion measurements: Spin echoes in the presence of a time-dependent field gradient. J. Chem. Phys. 1965, 42, 288-292.

5 Böhme, U.; Scheler, U. Effective charge of poly(styrenesulfonate) and ionic strength-an electrophoresis NMR investigation. Colloids Surf. A 2003, 222, 35-40.

6 Böhme, U.; Scheler, U. Counterion condensation and effective charge of poly(styrenesulfonate). Adv. Colloid Interf. Sci. 2010, 158, 63-67.

7 Böhme, U.; Hänel, B.; Scheler, U. Influence of the counterions on the behaviour of polyelectrolytes. Prog. Colloids Polym. Sci. 2011, in press.

8. Ballauff, M. Sperical polyelectrolyte brushes. Prog. Polym. Sci. 2007, 32, 1135-1151.

9. Hoffmann, M.; Jusufi, A.; Schneider, C.; Ballauff, M. Surface potential of spherical polyelectrolyte brushes in the presence of trivalent counterions. J. Colloid Interf. Sci. 2009, 338, 566-572. 
10. Böhme, U.; Scheler, U. Effective charge of bovine serum albumin determined by electrophoresis NMR. Chem. Phys. Lett. 2007, 435, 342-345.

11. Welch, P; Muthukumar, M. Tuning the density profile of dendritic polyelectrolytes. Macromolecules 1998, 31, 5892-5897.

12. Maiti, P.K.; Çağin, T.; Lin, S.T.; Goddard, W.A. Effect of solvent and $\mathrm{pH}$ on structure of PAMAM dendrimers. Macromolecules 2005, 38, 979-991.

13. Gurtovenko, A.A.; Lyulin, S.V.; Karttunen, M.; Vattulainen, I. Molecular dynamics study of charged dendrimers in salt-free solution: Effect of counterions. J. Chem. Phys. 2006, 124, 094904.

14 Klos, J.S.; Sommer, J.U. Simulations of terminally charged dendrimers with flexible spacer chains and explicit counterions. Macromolecules 2010, 43, 4418-4427.

15. Maiti, P.K.; Messina, R. Counterion distribution and $\zeta$ - potential in PAMAM dendrimer. Macromolecules 2008, 41, 5002-5006.

15. Cakara, D.; Kleimann, J.; Borkovec, M. Microscopic protonation equilibria of poly(amidoamine) dendrimers from macroscopic titrations. Macromolecules 2003, 36, 4201-4207.

16. Porcar, L.; Liu, Y.; Verduzco, R.; Hong K.; Butler, P.D.; Magid, L.J.; Smith, G.S.; Chen, W.R. Structural investigation of PAMAM dendrimers in aqueous solutions using small-angle neutron scattering: Effect of generation. J. Phys. Chem. B 2008, 112, 14772-14778.

17. Gomez, M.V.; Guerra, J.; Velders, A.H; Crooks, R.M. NMR characterization of fourth-generation PAMAM Dendrimers in the presence and absence of palladium dendrimer-encapsulated nanoparticles. J. Am. Chem. Soc. 2009, 131, 341-350.

(C) 2011 by the authors; licensee MDPI, Basel, Switzerland. This article is an open access article distributed under the terms and conditions of the Creative Commons Attribution license (http://creativecommons.org/licenses/by/3.0/). 